\title{
Cytosolic Proteome Profiling of Aminoglycosides Resistant Mycobacterium tuberculosis Clinical Isolates Using MALDI-TOF/MS
}

\author{
Divakar Sharma, Manju Lata, Rananjay Singh, Nirmala Deo, Krishnamurthy Venkatesan \\ and Deepa Bisht*
}

Department of Biochemistry, National JALMA Institute for Leprosy and Other Mycobacterial Diseases, Agra, India

OPEN ACCESS

Edited by:

Michael Thomas-Poulsen,

University of Copenhagen, Denmark

Reviewed by:

Jer-Horng Wu,

National Cheng Kung University,

Taiwan

Yixin Shi,

Arizona State University, USA

${ }^{*}$ Correspondence:

Deepa Bisht

abd1109@rediffmail.com

Specialty section:

This article was submitted to Antimicrobials, Resistance and

Chemotherapy,

a section of the journal

Frontiers in Microbiology

Received: 19 July 2016

Accepted: 28 October 2016

Published: 15 November 2016

Citation:

Sharma $D$, Lata $M$, Singh $R$, Deo $N$, Venkatesan K and Bisht D (2016) Cytosolic Proteome Profiling of Aminoglycosides Resistant

Mycobacterium tuberculosis Clinical Isolates Using MALDI-TOF/MS

Front. Microbiol. 7:1816.

doi: 10.3389/fmicb.2016.01816
Emergence of extensively drug resistant tuberculosis (XDR-TB) is the consequence of the failure of second line TB treatment. Aminoglycosides are the important second line anti-TB drugs used to treat the multi drug resistant tuberculosis (MDR-TB). Main known mechanism of action of aminoglycosides is to inhibit the protein synthesis by inhibiting the normal functioning of ribosome. Primary target of aminoglycosides are the ribosomal RNA and its associated proteins. Various mechanisms have been proposed for aminoglycosides resistance but still some are unsolved. As proteins are involved in most of the biological processes, these act as a potential diagnostic markers and drug targets. In the present study we analyzed the purely cytosolic proteome of amikacin (AK) and kanamycin (KM) resistant Mycobacterium tuberculosis isolates by proteomic and bioinformatic approaches. Twenty protein spots were found to have over expressed in resistant isolates and were identified. Among these Rv3208A, Rv2623, Rv1360, Rv2140c, Rv1636, and Rv2185c are six proteins with unknown functions or undefined role. Docking results showed that $A K$ and $K M$ binds to the conserved domain (DUF, USP-A, Luciferase, PEBP and Polyketidecyclase/dehydrase domain) of these hypothetical proteins and over expression of these proteins might neutralize/modulate the effect of drug molecules. TBPred and GPS-PUP predicted cytoplasmic nature and potential pupylation sites within these identified proteins, respectively. String analysis also suggested that over expressed proteins along with their interactive partners might be involved in aminoglycosides resistance. Cumulative effect of these over expressed proteins could be involved in AK and KM resistance by mitigating the toxicity, repression of drug target and neutralizing affect. These findings need further exploitation for the expansion of newer therapeutics or diagnostic markers against AK and KM resistance so that an extreme condition like XDR-TB can be prevented.

Keywords: amikacin, bioinformatic tools, kanamycin, cytosolic proteome, Mycobacterium tuberculosis, resistance 


\section{INTRODUCTION}

Tuberculosis (TB) still remains one of the deadliest communicable diseases worldwide which is caused by Mycobacterium tuberculosis. WHO reported 9.0 million people developed TB and 1.5 million deaths including 3,60,000 people with HIV (WHO Report, 2015). Widespread development of multidrug-resistant tuberculosis (MDR-TB) has worsened the circumstances and aminoglycosides are used to treat them. Failure of aminoglycosides anti-TB treatment leads to resistance as well as emergence of extensively drug resistant tuberculosis (XDR-TB). Aminoglycosides, amikacin (AK) and kanamycin $(\mathrm{KM})$ are important second line anti-mycobacterial drugs for MDR-TB patients. The principle targets of AK and KM resistance includes mutation in ribosomal protein/16S rRNA (Beauclerk and Cundliffe, 1987), enzymatic inactivation of drugs (Welch et al., 2005), decreased inner membrane transport and active efflux pumps (Magnet et al., 2001), cell wall impermeability (Nikaido, 2003), trapping of drug (Magnet et al., 2003). AK and KM resistance were contributed by rrs mutations in approximately $70 \% \mathrm{M}$. tuberculosis; however remaining 30\% does not have these mutations and signifying the contribution of some other resistance mechanism (s). Advancement in proteomics has cleared the doubts to prove any complex phenotypes. As proteins marked most of the biological processes, these are attractive targets for developing new drugs and diagnostics against the resistance. Two-dimensional gel electrophoresis (2-DE) coupled with MALDI-TOF-MS have direct approaches for separation, identification, and characterization of proteins and its species (Kumar et al., 2013; Sharma et al., 2015b). Comparative proteomic studies addressing whole cell lysate and membrane and membrane associated proteins of aminoglycosides resistance isolates have been reported (Kumar et al., 2013; Sharma et al., 2015b). Recently we have reported involvement of ferritin (a cytoplasmic protein) in $\mathrm{AK}$ and $\mathrm{KM}$ resistance (Sharma et al., 2016). However, purely cytosolic expression proteome of aminoglycosides resistant $M$. tuberculosis isolates have not been explored. To address this issue, we analyzed purely isolated cytosolic proteins of $\mathrm{AK}$ and $\mathrm{KM}$ resistant $M$. tuberculosis by direct proteomic and bioinformatics approaches. Such information could be helpful for the development of newer diagnostics and drug targets against $\mathrm{AK}$ and $\mathrm{KM}$ drug resistance so that the situations like extensively drug resistance could be prevented.

\section{MATERIALS AND METHODS}

\section{M. tuberculosis Isolates Collection and Drug Susceptibility Testing}

Four total susceptible (rifampicin, isoniazid, ethambutol, pyrazinamide, streptomycin, kanamycin, and amikacin) and four $\mathrm{AK}$ and $\mathrm{KM}$ resistant (sensitive to other first line and second line drugs) M. tuberculosis isolates were procured from Mycobacterial Repository Centre of National JALMA Institute for Leprosy and Other Mycobacterial Diseases, Agra, India. Drugs susceptibility testing (DST) for all the drugs was performed by LJ proportion (Canetti et al., 1969) and REMA methods (Palomino et al., 2002). Cultures were grown in Sauton's liquid medium at $37^{\circ} \mathrm{C}$ and harvested in late log phase (4 weeks) for proteomic analysis.

\section{Purely Cytosolic Proteins Isolation and Precipitation}

Mycobacterial cell lysate was prepared with slight modifications (Brodie et al., 1979; Sharma and Bisht, 2016). Briefly, Cells were suspended in sonication buffer with $1 \% \mathrm{v} / \mathrm{v}$ Triton $\mathrm{X}-100$ and then broken by intermittent sonication at $4^{\circ} \mathrm{C}$ for $20 \mathrm{~min}$. Homogenate was centrifuged at $12,000 \mathrm{~g}$ for $20 \mathrm{~min}$ at $4^{\circ} \mathrm{C}$. Resulting supernatants were ultracentrifuged at $150000 \mathrm{~g}$ for $90 \mathrm{~min}$. to obtain the purely cytosolic supernatant and the pellet (cell membrane) was discarded. Cytosolic supernatant was precipitated using published protocol (Bisht et al., 2007). Protein concentrations were estimated by Bradford method (Bradford, 1976). Protein extractions were performed for three times in biological and technical replicas.

\section{Two Dimensional Gel Electrophoresis}

IEF and SDS-PAGE were carried out using the published protocol of "in gel rehydration" with slight modifications (Gorg et al., 2000; Sharma and Bisht, 2016). In brief, IPG strips of $\mathrm{pH}$ 4-7 and length $17 \mathrm{~cm}$ (Bio-Rad, Hercules, CA, USA) were rehydrated overnight at $20^{\circ} \mathrm{C}$ with $550 \mu \mathrm{g}$ proteins. Strips were focused on an IEF unit (Bio-Rad) at $20^{\circ} \mathrm{C}$. Proteins were separated in second dimension on $12 \%$ SDS-polyacrylamide gels in a vertical electrophoresis unit PROTEAN II XI (BioRad). Gels were stained with Coomassie Brilliant Blue and analyzed using PDQuest Advanced software version 8.0.0 (BIORAD, Hercules, CA, USA). Protein spots which showed consistently increased intensities with more than 1.5 fold were selected for identification. Student t-test (inbuilt with software) was used for the statistical analysis by PDQuest Advanced software. The system picks up the spots with differential intensity of significant levels built in the system. Equal amount of protein was loaded in all gels and experiments were repeated in biological and technical replicates for at least three times.

\section{In Gel Digestion and Mass Spectrometry}

In-gel digestion of proteins and MALDI-TOF/MS was carried out using published protocol (Shevchenko et al., 1996; Sharma et al., 2015b). In brief, mass spectra of digested proteins were acquired using Autoflex II TOF/TOF 50 (Bruker Daltonik GmbH, Leipzig, Germany). PMF were submitted to swissprot database for their identification taking taxonomy as $M$. tuberculosis complex. Peptide mass tolerance was set in range of 50-125 ppm (to acquire the best MASCOT score) with carbamidomethyl-cystein set as fixed modification. The oxidation of methionine was set as variable modification and one missed cleavage site was allowed. 


\section{Bioinformatic Analysis}

Protein sequences of hypothetical proteins were retrieved from Tuberculist server http://tuberculist.epfl.ch/. Classes of proteins were predicted using TBpred server (Rashid et al., 2007). There probable functions and interacting partners were predicted using InterProScan, Molecular docking (Table 3), GPS-PUP (Quevillon et al., 2005; Schneidman-Duhovny et al., 2005; Andrusier et al., 2007; Mashiach et al., 2008; Liu et al., 2011) and STRING analysis (Search Tool for the Retrieval of Interacting Genes/Proteins, http://string.embl.de/) (Mawuenyega et al., 2005). STRING-10, server was used to predict the interacting partners of protein-protein interaction. STRING database uses a combination of prediction approaches and an integration of other information (neighborhood, transferred neighborhood, gene fusion, co-occurrence, coexpression, experiments, databases, text mining). Network was made at medium confidence level (0.400) allowing all active prediction methods.

\section{RESULTS}

In this study we have compared the cytosolic protein profiles of $\mathrm{AK}$ and $\mathrm{KM}$ resistant (lack rrs mutation) with total sensitive isolates. $2 \mathrm{DE}$ was run in triplicates for all isolates and composite images are shown in Figure 1. Comparison of 2D gels by PDQuest Advanced software revealed twenty protein spots which were consistently over expressed in resistant as compared to sensitive isolates (cut limit $\geq 1.5$ fold changes in spot intensity). For statistical analysis inbuilt student $t$-test was used by PDQuest advanced software. Over expressed proteins spots were identified by MALDITOF-MS (Table 1). The identified proteins were Trigger factor
(Rv2462c), 3-oxoacyl-[acyl-carrier-protein] synthase II (Rv2246), 3-ketoacyl-ACP reductase (Rv0242c), Transcriptional regulator MoxR1(Rv1479), Fructose-bisphosphate aldolase (Rv0363c), Universal stress protein (Rv2623), Uncharacterized Protein (Rv1360), Alkyl hydroperoxide reductase subunit C (Rv2428), Enoyl-coA hydratase (Rv0632c), Adenylate kinase (Rv0733), Single stranded DNA-binding protein (Rv0054), Peptidalprolyl isomerase (Rv0009), Beta carbonic anhydrase 1(Rv1284), UPF0098 protein (Rv2140c), Hypothetical protein (Rv2185c), Universal stress protein (Rv1636), Alpha-crystallin/HspX (Rv2031c), Probable cold shock protein A (Rv3648c), and Conserved Hypothetical protein (Rv3208A). However, two spots were identified as a protein of its species and therefore total 19 proteins were found. Among these Rv2623, Rv1360, Rv2140c, Rv2185c, Rv1636, and Rv3208A were proteins of unknown function. Out of nineteen, Rv0363c, Rv1360, Rv0733, and Rv1284 belonged to intermediary metabolism and respiration, Rv2623, Rv2428, and Rv1636, Rv2031c, and $\mathrm{Rv} 3648 \mathrm{c}$ to virulence/detoxification/adaptation, Rv2462c to cell wall and cell processes, Rv2246, Rv0242c, and Rv0632c to lipid metabolism, Rv2140c, Rv2185c and Rv3208A to conserved hypothetical, Rv1479 to regulatory proteins and Rv0054 and Rv0009 to information pathway categories. The level of difference in protein spot intensity has been represented as densitometric ratio in Table $\mathbf{1}$. These proteins were also reported in cytosolic/whole cell lysate fraction of M. tuberculosis complex by various authors [23-26].

\section{TBPred}

TBPred analysis revealed that most of the identified proteins were found to be predicted in the class of cytoplasmic proteins (Table 2).
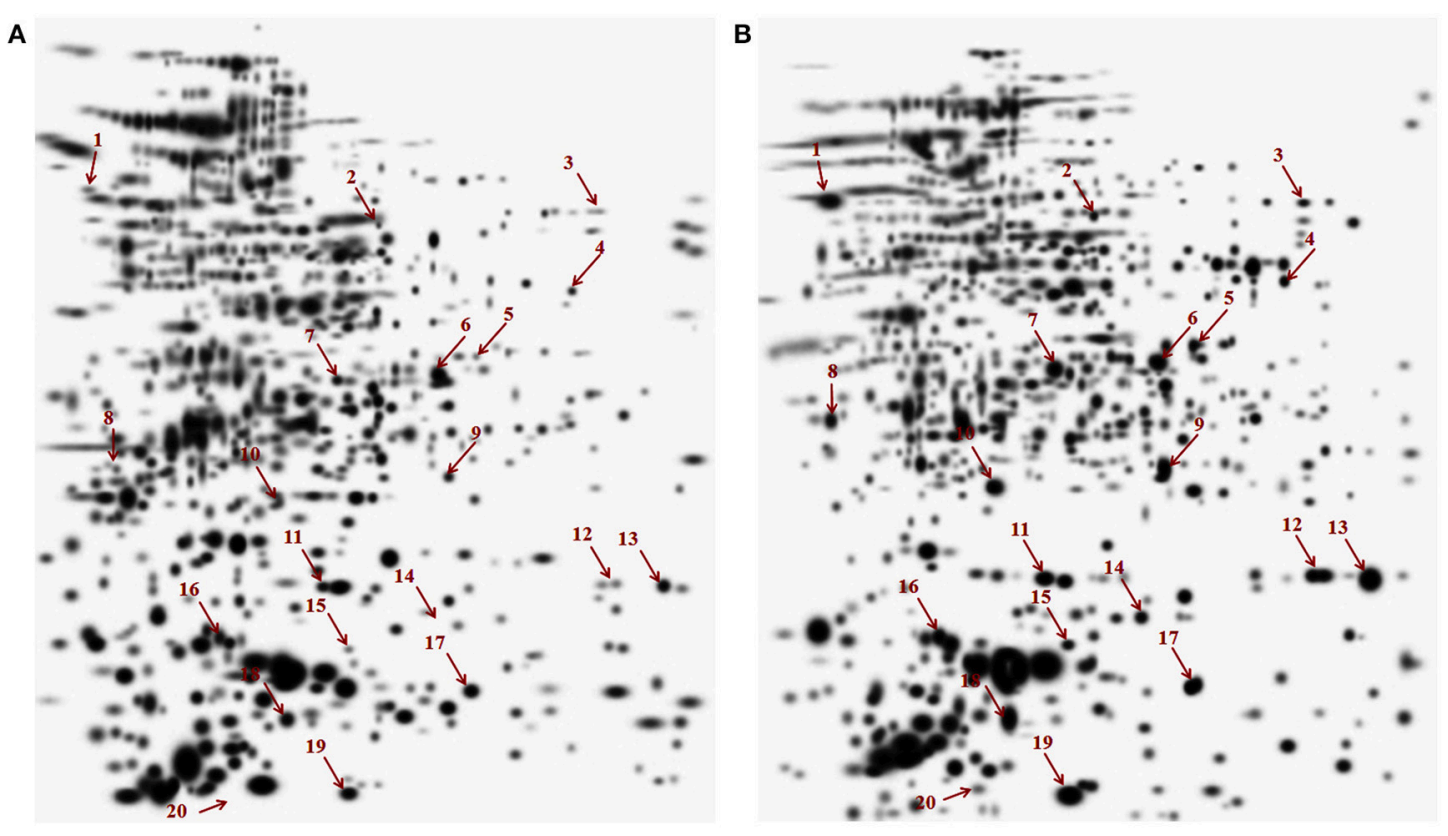

FIGURE 1 | Composite images of 2DE profile of cytosolic proteome of M. tuberculosis isolates (A) Total susceptible (B) AM and KM resistant isolates. 
TABLE 1 | Details of proteins identified by mass spectrometry.

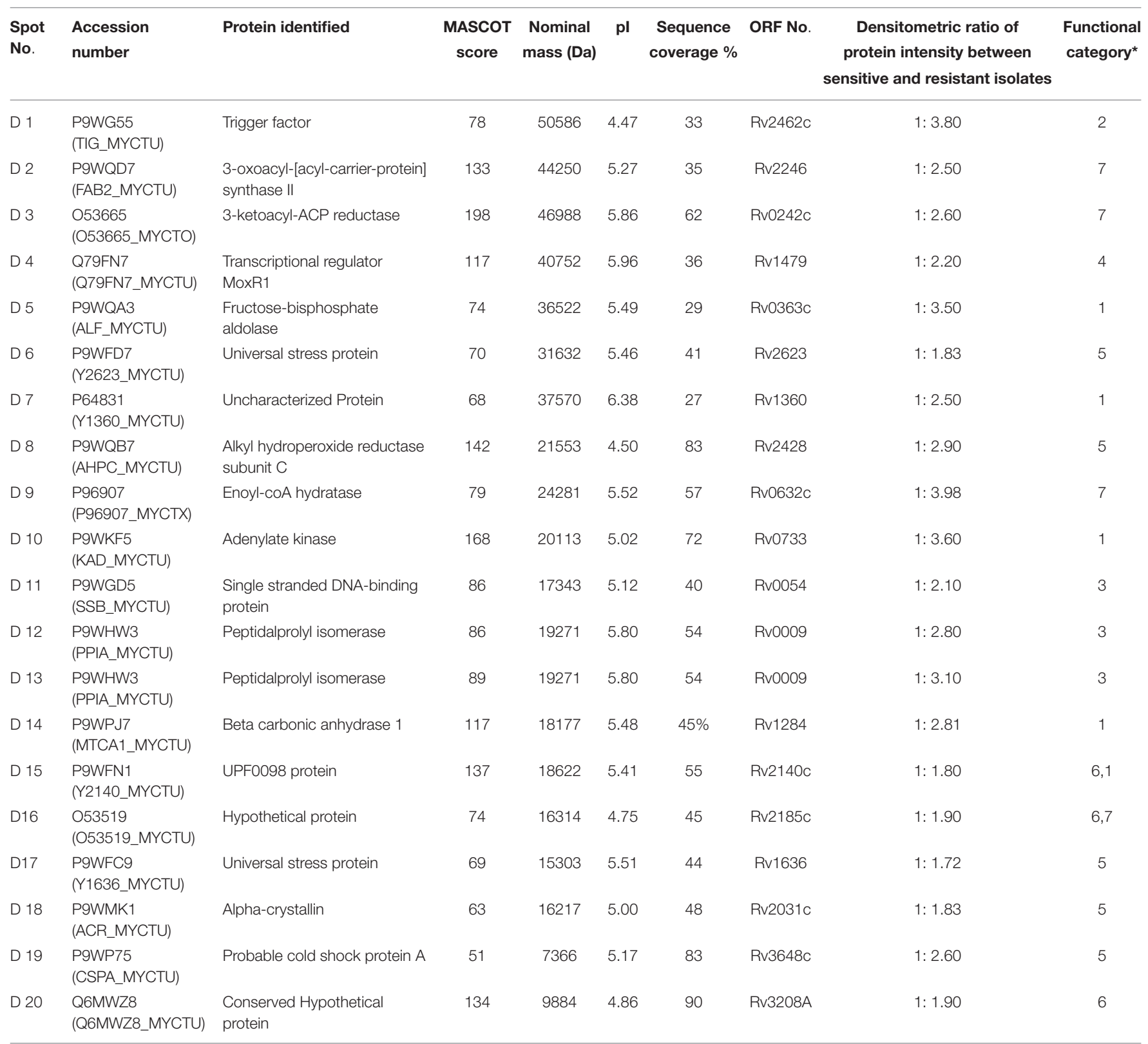


hypothetical; 7, lipid metabolism.

*Note on functional category.

\section{Interproscan Analysis}

InterProScan analysis of Rv3208A showed the presence of conserved domain (DUF3107) of protein of unknown function (\#PF11305) from residues 1-73. Rv2623 (Universal stress protein) was found to be highly conserved in mycobacterial species and it appeared as hypothetical protein with unknown function. InterProScan analysis of Rv2623 showed the presence of two conserved USP-A domain with amino acid residues from 10 to 148 and 162-293 (\#PF00582). Rv1360 showed conserved Luciferase like domain from residues 15-316 (\#PF00296) and Rv2140c showed conserved Phosphatidylethanolamine binding protein (PEBP) domain (\#SSF49777) from residues 17-174. Rv1636 showed the presence of conserved UspA domain with amino acid residues from 4 to 144 (\#PF00582). Rv2185c showed the presence of Polyketide cyclase/dehydrase domain with amino acid residues from 4 to 143 (\#PF10604).

\section{D Modeling and Docking}

Molecular docking analysis of selected 3D models (showing less than $2 \%$ discrepancy from Ramachandran plot) of hypothetical proteins was performed to find out their binding sites with $\mathrm{AK}$ and KM. Parameters used for selection of 3D models 
TABLE 2 | Prediction of classes of these identified proteins by TBpred server.

\begin{tabular}{|c|c|c|c|}
\hline ORF No. & $\begin{array}{c}\text { Score of amino acid composition } \\
\text { based SVM approach }\end{array}$ & $\begin{array}{l}\text { Score of dipeptide composition } \\
\text { based SVM approach }\end{array}$ & Final predicted class of proteins \\
\hline Rv2462c & 3.108 & 1.355 & Cytoplasmic \\
\hline Rv2246 & 0.927 & 0.949 & Cytoplasmic \\
\hline Rv0242c & 0.704 & -0.009 & Integral MP/Cytoplasmic \\
\hline Rv1479 & 2.402 & 0.057 & Integral MP \\
\hline Rv0363c & 2.062 & 2.189 & Cytoplasmic \\
\hline Rv2623 & 1.061 & 0.549 & Cytoplasmic/Integral MP \\
\hline$R v 1360$ & 1.226 & 0.616 & Cytoplasmic \\
\hline Rv2428 & 0.417 & 0.460 & Cytoplasmic \\
\hline Rv0632c & 0.676 & 0.482 & Integral MP/Cytoplasmic \\
\hline Rv0733 & 4.242 & 2.154 & Cytoplasmic \\
\hline Rv0054 & 0.319 & -0.496 & Attached to Membrane by Lipid Anchor/Secretory \\
\hline Rv0009 & 0.553 & 0.223 & Attached to Membrane by Lipid Anchor \\
\hline Rv1284 & 1.762 & 1.515 & Cytoplasmic \\
\hline Rv2140c & 0.110 & 0.081 & Integral MP/Cytoplasmic \\
\hline Rv2185c & 3.738 & 2.543 & Cytoplasmic \\
\hline Rv1636 & 3.225 & 0.341 & Cytoplasmic \\
\hline Rv2031c & 2.524 & 2.397 & Cytoplasmic \\
\hline Rv3648c & 0.430 & 1.765 & Integral MP/Cytoplasmic \\
\hline Rv3208A & 2.287 & 1.209 & Cytoplasmic \\
\hline
\end{tabular}

and molecular docking are represented in Table 3. Docking of Rv3208A (Figure 2) showed the interaction of AK and KM drugs to the conserved motif of domain of unknown function (DUF3107 domain). Docking with Rv2623 showed that both drugs bind to the residues of central cavity of USP-A domain of universal stress protein (Figure 2). For both drugs interacting residues were almost common, which suggests similar binding sites. Docking with Rv1360 (Figure 3) showed that both drugs bind to the interacting residues of conserved Luciferase like domain of the uncharacterized protein. Docking with Rv2140c showed that both drugs interacted with amino acids residues of conserved PEBP domain of hypothetical UPF0098 protein (Figure 3). Docking analysis of Rv1636 and Rv2185c (Figure 4) showed that AK and KM binds to conserved USP-A domain of uncharacterized protein and conserved Polyketide cyclase/dehydrase domain of hypothetical protein, respectively.

\section{Prediction of Pupylation Sites}

Using the default threshold (medium) with cutoff 2.452, GPSPUP predicted pupylation sites in 15 identified proteins which are tabulated in Table 4. Four proteins have not shown any pupylation sites with these default parameters.

\section{String Analysis}

We analyzed the 19 over expressed proteins using STRING10 with a medium confidence score threshold of 0.4 and build an interactome network (Figure 5) for these set of proteins to find out the protein-protein interaction (PPIs) and predict functional associations. We found that proteins involved in intermediary metabolism and respiration, lipid metabolism, information pathway/regulatory, and virulence, detoxification and adaptation category interacted with each other as well as their partners except the hypothetical proteins.

\section{DISCUSSION}

In this study we analyzed the comparative cytosolic proteome of $\mathrm{AK}$ and $\mathrm{KM}$ resistant isolates by 2DE-MALDI-TOF/MS and bioinformatic tools. Over expressed proteins in the resistant clinical isolates were identified, which may be used as diagnostic markers or drug targets for therapeutics. 2DE/MS has an advantage over the traditional methods (SDSPAGE, chromatography and sequencing) as not only the identification of a large number of unknown proteins but also protein species separation. Several reports (whole cell lysates and membrane and membrane associated proteins) on aminoglycosides drug resistance and identification of diagnostics/drug targets employing proteomic and bioinformatic approaches exists (Sharma et al., 2010a, 2014, 2015a,b, 2016; de Souza et al., 2011; Kumar et al., 2013) which suggested that over expressed hypothetical proteins (Rv3867, Rv3224, Rv0148,Rv2744c), universal stress protein (Rv2005c) and some known proteins (involved in various pathways) might be involved in aminoglycosides resistance. However, to the best of our knowledge, no study on cytosolic proteome analysis with AK and KM resistant M. tuberculosis isolates has been reported.

\section{Repression of Targets}

Rv0363c (Fructose bisphosphate aldolase) is centrally involved in glycolysis, gluconeogenesis, and ATP synthesis. Mycobacteria reside in specialized niches and may require adaptations in the energy metabolism. It is essential for growth/pathogenesis of 
TABLE 3 | Modeling, docking parameters and analysis of interacting amino acids to drugs.






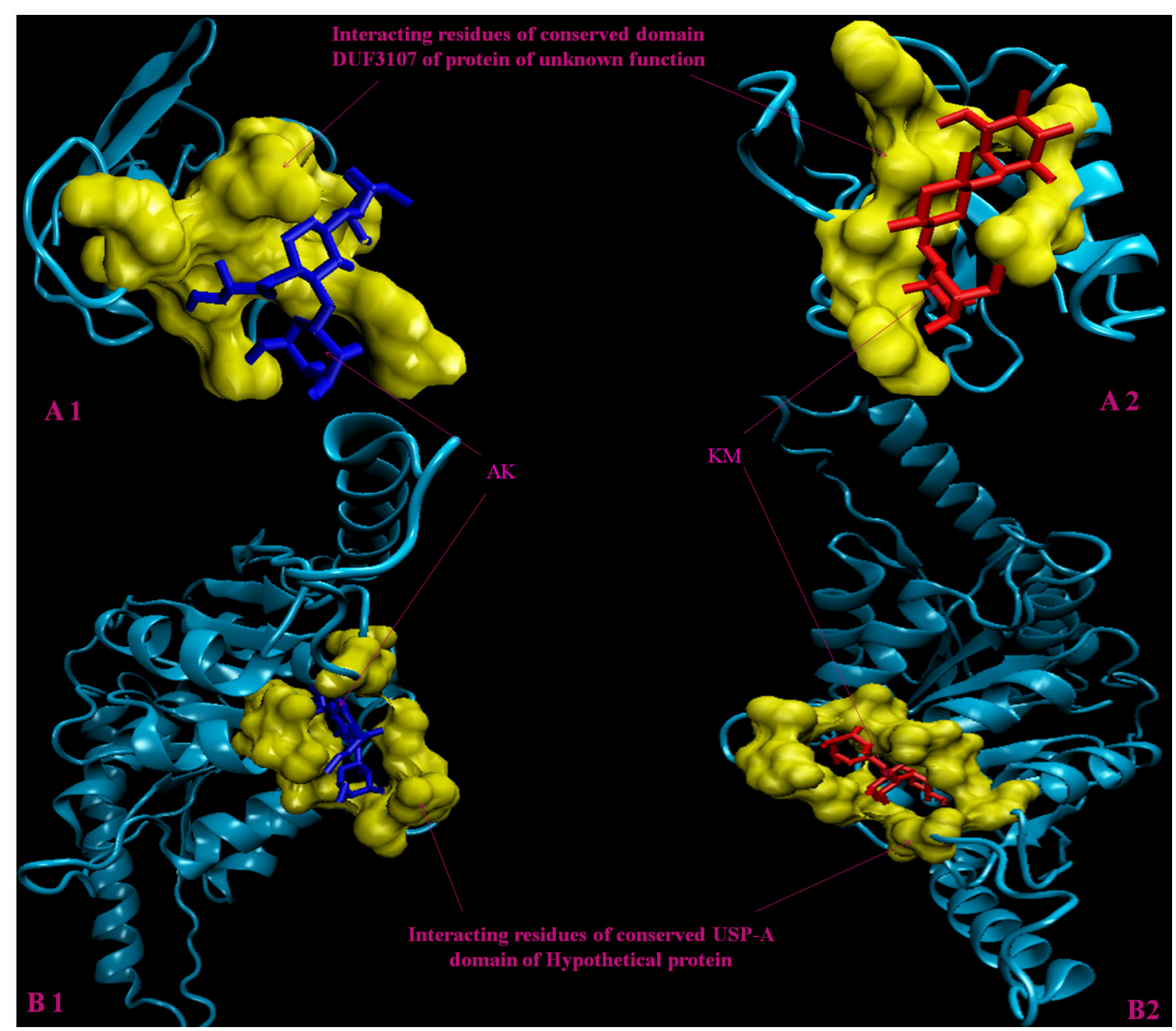

FIGURE 2 | 3D model of hypothetical proteins showing docking with AK and KM. (A1,A2) shows molecular docking of Rv3208A with AM (blue) and KM (red), respectively, yellow color shows interacting residues of DUF3107 domain. (B1,B2) shows molecular docking of Rv2623 with AM (blue) and KM (red), respectively, yellow color show interacting residues of USP-A domain.

replicating and dormant $M$. tuberculosis (de la Paz Santangelo et al., 2011). Puckett et al. suggested that it regulates glycolytic and gluconeogenic carbon co-catabolism in $M$. tuberculosis (Puckett et al., 2014). Aminoglycosides resistant mycobacteria also behave like dormant bacilli due to repression of targets. Low translational state is an intrinsic defense mechanism of aminoglycosides resistant mycobacteria. Over expression of $\mathrm{Rv} 0363 \mathrm{c}$ might maintain energy supply to resistant M. tuberculosis. Rv0733 (Adenylate kinase) is a small ubiquitous enzyme involved in ATP metabolism. Meena et al. suggested that Rv0733 has dual activity as nucleoside mono-and diphosphate kinase and could be implicated in the purine salvage pathway (Meena et al., 2003). Recently Lata et al. reported that Rv0733 is over expressed in ofloxacin mono resistant $M$. tuberculosis (Lata et al., 2015b). Over expression of Rv0733 maintains nucleotide supply to cells even in low translational state or dormant state and act as attractive targets for the development of new antimycobacterial agents. Rv1284 ( $\beta$-carbonic anhydrase) catalyzes the reversible hydration of carbon dioxide to form bicarbonate and is also needed for fatty acid biosynthesis as well as $\mathrm{pH}$ homeostasis, therefore it is considered as essential for survival of M.tuberculosis. Rv1284 gene was highly over expressed under nutrients starved condition (Betts et al., 2002). Nienaber et al. reported Tyr120 of Rv1284 is critical residue for oxidative inactivation and its involvement in $\mathrm{pH}$ homeostasis/ redox-regulation (Nienaber et al., 2015). Over expression of Rv1284 might be involved in pathogenesis by maintaining the homeostasis/biosynthesis of fatty acids. Rv1479 (Transcriptional regulator MoxR1) is involved in regulatory function. $\mathrm{Hu}$ and Coates reported that its m-RNA was 4 -fold over expressed in persisters as compared to stationary phase of mycobacteria (Hu and Coates, 2001). Recently Sharma et al. reported it's over expression in aminoglycosides resistant $M$. tuberculosis (Sharma et al., 2015b). Aminoglycosides resistant mycobacteria also behave like dormant bacilli due to repression of targets which is the mycobacterial intrinsic defense mechanism 


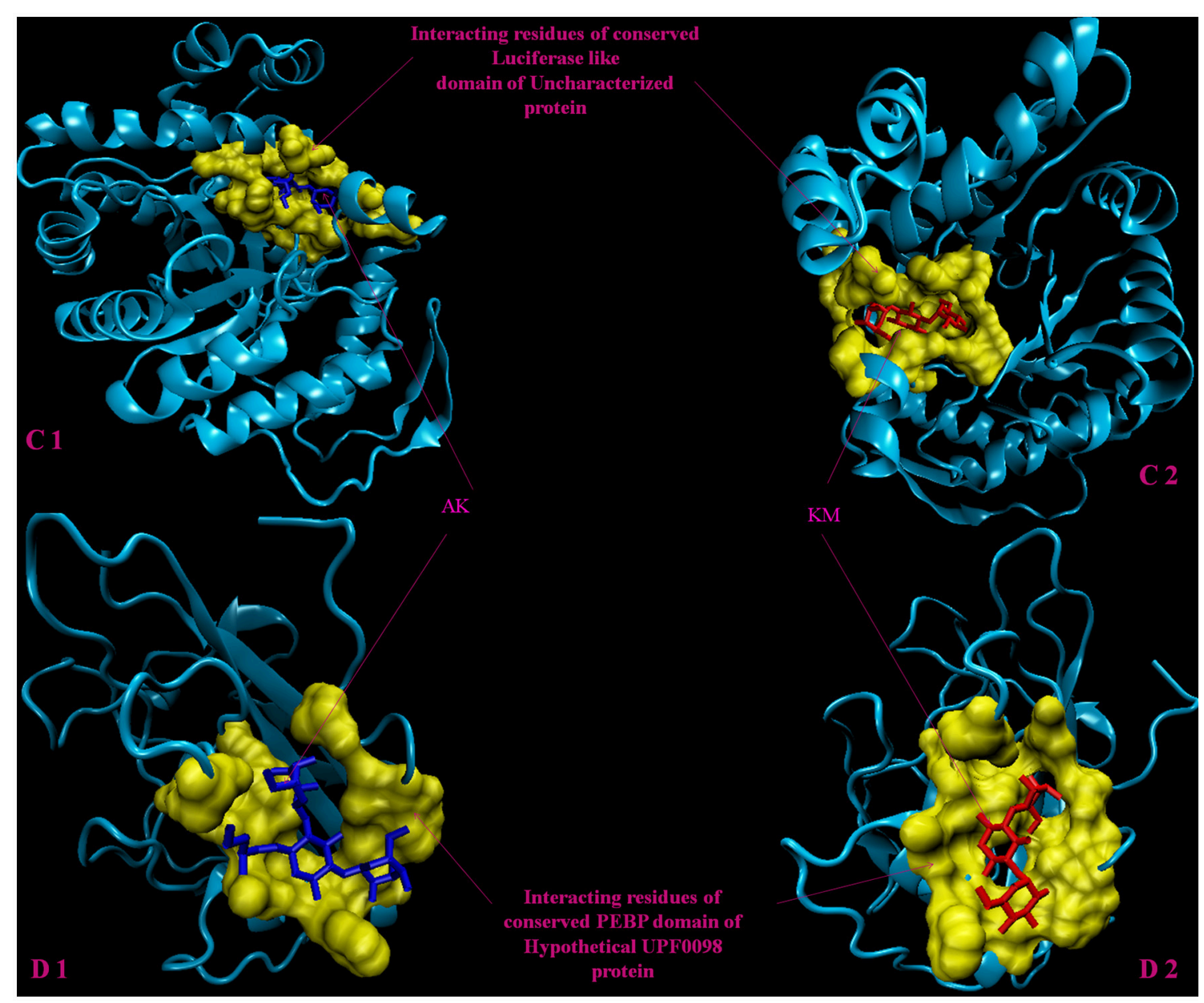

FIGURE 3 | 3D model of hypothetical proteins showing docking with AK and KM. (C1,C2) shows docking of Rv1360 with AM (blue) and KM (red), respectively, yellow color shows interacting residues of luciferase like domain. (D1,D2) shows docking of Rv2140c with AM (blue) and KM (red), respectively, yellow color shows interacting residues of conserved PEBP domain of hypothetical UPF0098 protein.

against resistance. Our study assumes that over expression of this protein regulate the transcription of mycobacteria even in low transcriptional state or dormant state. Rv0009 (Probable iron-regulated peptidyl prolyl cis-trans isomerase A) is probably involved in cis-trans isomerization of proline imidic peptide bonds in oligopeptides and accelerated protein folding (Henriksson et al., 2004). Recently Lata et al. reported the higher expression of Rv0009 in ofloxacin and moxifloxacin induced M. tuberculosis (Lata et al., 2015a). Over expression of Rv0009 might maintain cis-trans isomerization of amino acids and its supply to cells even in low translational state or dormant state.

We hypothesized that over expression of Rv0363c, Rv0733, Rv1284, Rv1479, and Rv0009 proteins might cumulatively overcome the effect of targets repression and contribute to aminoglycosides resistance.

\section{Mitigate the Toxicity}

Rv2462c (trigger factor, also acts as chaperone), is not only involved in protein export but also maintains the open conformation of newly synthesized protein. It was reported that its intensity is regulated by phosphate depletion (Rifat et al., 2009). Sharma et al. reported that it was over expressed in aminoglycosides resistant $M$. tuberculosis (Sharma et al., 2015b). Over expression of Rv2462c might mitigate the toxicity by maintaining the export of newly synthesized protein or truncated proteins in an open conformation. Rv2031c (Alphacrystallin/HspX), protect the cells from different stimuli like stress, dormancy, drug and hypoxia by preventing protein aggregation (Sherman et al., 2001). In our previous studies, increased intensity of Rv2031c was reported in resistant M. tuberculosis (Kumar et al., 2013; Sharma et al., 2015b). Over expression of Rv2031c might mitigate the toxicity by preventing protein aggregation. Rv3648c (probable cold shock protein A) possibly involved in cold acclimation processes like dormancy, stress, drug and hypoxia (Hu et al., 1999). Rv3648c mRNA showed five to sevenfold increase in Mycobacterium smegmatis under cold-induction dormancy (Shires and Steyn, 2001). Over expression of Rv3648c might mitigate the aminoglycosides toxicity by maintaining the protein conformation. Rv2428 


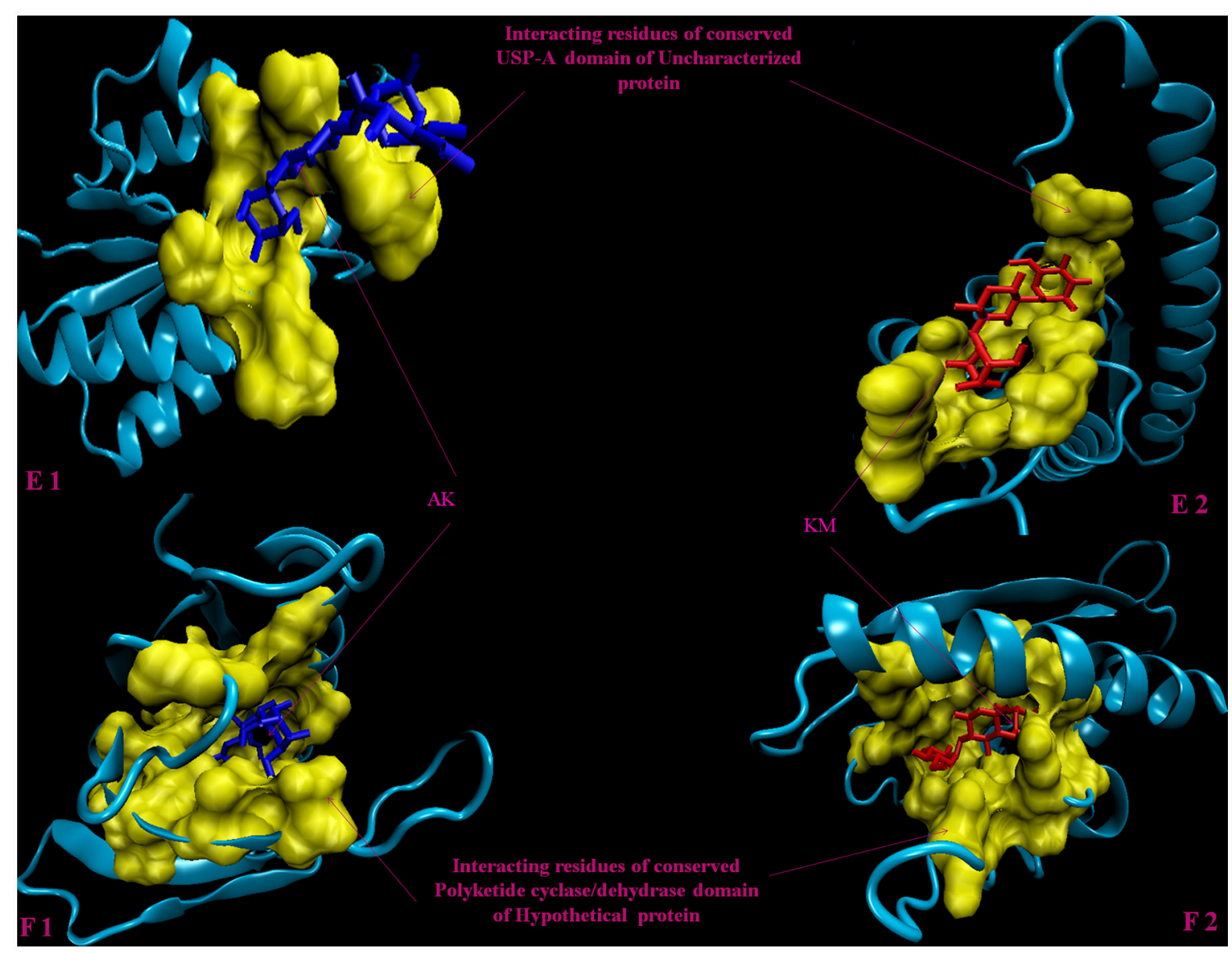

FIGURE 4 | 3D model of hypothetical proteins showing docking with AK and KM. (E1,E2) shows docking of Rv1636 with AM (blue) and KM (red), respectively, yellow color shows interacting residues of conserved USP-A domain, (F1,F2) shows docking of Rv2185c with AM (blue) and KM (red), respectively, yellow color shows interacting residues of conserved Polyketide cyclase/dehydrase domain of hypothetical protein.

(Alkyl hydroperoxide reductase $\mathrm{C} / \mathrm{AhpC}$ ), a novel group of the peroxiredoxin family that reduces organic peroxide and hydrogen peroxides. Heym et al. reported that over expression of the AhpC involved in virulence and isoniazid resistance of M. tuberculosis (Heym et al., 1997). Lee et al. suggested that AhpC expression was induced under oxidative stress conditions and involved in M. smegmatis (Lee et al., 2014). Over expression of Rv2428 might mitigate the toxic effects of the aminoglycosides. Rv0054 (SS-DNA binding protein/SSB) involved to maintain DNA/RNA integrity in replication, recombination and repair phenomena. SSBs are also involved in modulating the activity of DNA polymerase, RNA polymerase and DNA helicase. Over expression of Rv0054 was found in drug induced study (Lata et al., 2015a). Over expression of Rv0054 might mitigate the toxicity/stress of drugs. Rv2246 (3-oxoacyl-[acyl-carrier protein] synthase 2) was involved in meromycolate elongation step of mycolic acid biosynthetic pathway (Singh et al., 2011) which provides a thick layer of lipid in the cell wall and protects $M$. tuberculosis from environmental and poisonous chemicals stress. Starck et al. reported that in M. tuberculosis Rv2246 was over expressed under anaerobic conditions (Starck et al.,
2004). Over expression of Rv2246 might mitigate the toxicity of aminoglycosides. Rv0242c (Probable 3-oxoacyl-[acyl-carrier protein] reductase/FabG4) is involved in the first step of fatty acid biosynthesis pathway. Beste et al. suggested fabG4 to be essential for growth of M. bovis BCG on Roisin's medium (Beste et al., 2009). Sharma et al. suggested that Rv0242c might play a role in altering the drug permeability in resistant isolate by changing the fatty acid composition of the cell envelope (Sharma et al., 2010a). Over expression of Rv0242c might mitigate the toxicity of aminoglycosides. Rv0632c (Probable enoyl-CoA hydratase/EchA3) could possibly oxidize fatty acids. Maurya et al. reported that Rv0632c was overexpressed under anaerobic culture condition (Maurya et al., 2014). Recently Vargas-Romero et al. suggested that over expression of Rv0632c in hyper virulent M. tuberculosis CPT31 could facilitate mycobacterial infection and persistence (Vargas-Romero et al., 2016). Over expression of Rv0632c might mitigate the toxic effects of aminoglycosides.

We hypothesized that over expression of Rv2462c, Rv2031c, Rv3648c, Rv2428, Rv0054, Rv2246, Rv0242c, and Rv0632c proteins might cumulatively mitigate the toxicity and contribute to aminoglycosides resistance. 
TABLE 4 | Predicted/identified pupylation sites within identified proteins.

\begin{tabular}{|c|c|c|c|c|}
\hline ORF No. & $\begin{array}{c}\text { Position of } \\
\text { lysine residue } \\
\text { undergoes pupylation }\end{array}$ & Peptides & Score & Cut-off \\
\hline \multirow[t]{4}{*}{ Rv2462c } & 2 & ${ }^{* \star \star \star \star \star \star V K S T V E Q L S ~}$ & 2.614 & 2.452 \\
\hline & 33 & PDFQRAYKELAKQVR & 3.394 & 2.452 \\
\hline & 274 & SDQVRQAKRAQQAEQ & 2.669 & 2.452 \\
\hline & 439 & DTSEFFGKRVSAGEA & 2.677 & 2.452 \\
\hline \multirow[t]{2}{*}{ Rv2246 } & 54 & TDAETTWKLLLDRQS & 2.543 & 2.452 \\
\hline & 164 & VSPLTVQKYMPNGAA & 2.795 & 2.452 \\
\hline \multirow[t]{4}{*}{ Rv0242c } & 100 & ITEPAGLKGLHEFFT & 2.591 & 2.452 \\
\hline & 150 & GFTRSLGKELRRGAT & 2.472 & 2.452 \\
\hline & 168 & VYLSPDAKPAATGLE & 3.827 & 2.452 \\
\hline & 381 & LAPGLAAKGITINAV & 3.323 & 2.452 \\
\hline Rv1479 & 71 & MLVGLLSKGHVLLEG & 2.646 & 2.452 \\
\hline \multirow[t]{4}{*}{ Rv0363c } & 149 & AIAQELLKAAAAAKI & 3.669 & 2.452 \\
\hline & 155 & LKAAAAAKIILEIEI & 3.016 & 2.452 \\
\hline & 189 & TSPEDFEKTIEALGA & 3.165 & 2.452 \\
\hline & 318 & YDPRSYLKKAEASMS & 2.52 & 2.452 \\
\hline Rv2623 & 35 & ARDAELRKIPLTLVH & 3.63 & 2.452 \\
\hline \multirow[t]{3}{*}{ Rv2428 } & 25 & LIGGDLSKVDAKQPG & 4.071 & 2.452 \\
\hline & 70 & TEIAAFSKLNDEFED & 3.11 & 2.452 \\
\hline & 192 & LDAGELLKASA ${ }^{\star \star \star *}$ & 6.024 & 2.452 \\
\hline \multirow[t]{3}{*}{ Rv0733 } & 23 & QAVKKLAEKLGIPQIS & 4.26 & 2.452 \\
\hline & 94 & PRSVEQAKALHEMLE & 5.756 & 2.452 \\
\hline & 181 & RALRALGK $\mathbf{K}^{\star \star \star \star \star \star \star}$ & 5.866 & 2.452 \\
\hline \multirow[t]{2}{*}{ Rv0054 } & 84 & VIVSGRLKQRSFETR & 2.866 & 2.452 \\
\hline & 95 & FETREGEKRTVIEVE & 2.945 & 2.452 \\
\hline \multirow[t]{2}{*}{ Rv0009 } & 133 & QFFITVGKTPHLNRR & 2.646 & 2.452 \\
\hline & 161 & RWEAISKTATDGND & 2.772 & 2.452 \\
\hline Rv2140c & 133 & QFFITVGKTPHLNRR & 2.646 & 2.452 \\
\hline \multirow[t]{4}{*}{ Rv2185c } & 89 & LESSSLLKSLEGTYR & 3.079 & 2.452 \\
\hline & 136 & RLIDGALKDLKKRVE & 3.236 & 2.452 \\
\hline & 139 & DGALKDLKKRVEG** & 2.803 & 2.452 \\
\hline & 140 & GALKDLKKRVEG*** & 3.496 & 2.452 \\
\hline \multirow[t]{5}{*}{ Rv2031c } & 64 & LPGVDPDKDVDIMVR & 2.669 & 2.452 \\
\hline & 85 & KAERTEQKDFDGRSE & 2.748 & 2.452 \\
\hline & 114 & GADEDDIKATYDKGI & 3.386 & 2.452 \\
\hline & 119 & DIKATYDKGILTVSV & 2.913 & 2.452 \\
\hline & 132 & SVAVSEGKPTEKHIQ & 3.433 & 2.452 \\
\hline \multirow[t]{2}{*}{ Rv3648c } & 47 & RTLEENQKVEFEIGH & 3.236 & 2.452 \\
\hline & 57 & FEIGHSPKGPQATGV & 2.591 & 2.452 \\
\hline Rv3208A & 85 & AAAGSAGKVATSG** & 4.126 & 2.452 \\
\hline
\end{tabular}

Bold letter "K" means it undergoes pupylation.

*It is the blank space where no amino acid occurs.

\section{Neutralizing Effect}

Rv2623 and Rv1636 are universal stress proteins with unknown function. These were over-expressed under nutrient/oxygen limitation and be involved in virulence/chronic infection (Stewart et al., 2001). Recently Lata et al. reported that Rv2623 and Rv1636 were over expressed in ofloxacin and moxifloxacin induced study (Lata et al., 2015a). In the present study we have observed that $\mathrm{AK}$ and $\mathrm{KM}$ interacted to the residues of conserved USP-A domains of Rv2623 and Rv1636, which might be altering the functions. It is suggested that over expression of these proteins might neutralize/compensate the effect of drugs. Rv1360 (probable oxidoreductase) is an uncharacterized protein, probably involved in cellular metabolism. Kruh et al. reported that Rv1360 was expressed in guinea pig model during early and chronic stages of disease (Kruh et al., 2010). The bacteria are thought to be in a state of reduced replication and metabolism as part of the chronic lung infection as well as in aminoglycosides resistance; therefore over expression of Rv1360 might maintain the replication and metabolism. Through in-silico approaches Raman and Chandra suggested that it is a potential drug target against tuberculosis (Raman and Chandra, 2008). In the present study we have observed that $\mathrm{AK}$ and $\mathrm{KM}$ interacted to the residues of conserved Luciferase like domain, which might be altering the functions. Rv2140c (UPF0098 protein/conserved hypothetical protein/TB18.6) is the protein of unknown function. It is reported that Rv2140c had a phosphatidyl ethanolaminebinding protein (Eulenburg et al., 2013). Recently Lata et al. reported that Rv2140c was over expressed in ofloxacin resistant M. tuberculosis (Lata et al., 2015b). In the present study we have observed that $\mathrm{AK}$ and $\mathrm{KM}$ interacted to the residues of conserved Phosphatidylethanolamine binding protein (PEBP) domain, which might be altering the functions. Rv2185c (Conserved hypothetical protein/TB16.3) is the protein of unknown function. Starck et al. reported that in M. tuberculosis Rv2185c was over expressed under anaerobic conditions (Starck et al., 2004). It is reported that over expression of Rv2185c

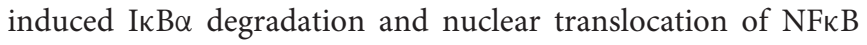
(Zhang et al., 2015). In the present study we have observed that AK and KM interacted to the residues of conserved Polyketide cyclase/dehydrase domain, which might alter the function. Rv3208A (Conserved hypothetical protein/TB 9.4) is the protein of unknown function. Griffin et al. suggested that expression of Rv3208A is essential for M. tuberculosis growth and cholesterol catabolism (Griffin et al., 2011). In our study we have found that AK and KM interacted to the residues of conserved DUF3107 domain, which might alter the function.

We hypothesized that over expression of Rv2623, Rv1636, Rv1360, Rv2140c, Rv2185c, and Rv3208A hypothetical proteins might cumulatively neutralize/compensate the effect of drugs and contribute to aminoglycosides resistance.

Pupylation is a reversible PTM which is likely to have a regulatory role (Burns et al., 2010). Pupylation contributes to the virulence/survival strategy of $M$. tuberculosis in the host and makes the bacteria more resistant to drug and other stresses (Darwin et al., 2003). In our study, out of 19 over expressed proteins 15 showed the pupylation sites which suggested that over expression of these proteins via 




FIGURE 5 | STRING analysis reveals the interaction partners of the over expressed proteins which showed that over expressed proteins interacted to proteins of the similar functional categories except the hypothetical proteins.

Pup-proteasome system (protein-protein interaction) might be involved in proteins turnover to overcome aminoglycosides stress. Interactome revealed that overexpressed proteins of lipid metabolism (LM), intermediary metabolism and respiration (IMR), information pathway/regulatory proteins (IP/RP), and virulence, detoxification and adaptation (VDA) category interacted to the other proteins which were involved in pathways of LM, IMR, IP/RP and VDA categories. We suggested that over expressed proteins along with their interactive partners of these various functional categories might cumulatively be involved in aminoglycosides resistance.

We assume that over expressed proteins might be contributing in $\mathrm{AK}$ and $\mathrm{KM}$ resistance and might act as diagnostic marker or potential targets for drug development against resistance.

\section{CONCLUSION}

In a nutshell, in this report we focus on the purely cytosolic proteins of $\mathrm{AK}$ and $\mathrm{KM}$ resistant $M$. tuberculosis using proteomic and bioinformatic approach. Among the 19 over expressed proteins, 13 with their defined roles and six with unknown functions. TBpred predicted most of the proteins belong to cytoplasmic class. Molecular docking of these six hypothetical proteins (Rv2623, Rv1636, Rv1360, Rv2140c, Rv2185c, and Rv3208A) showed that AK and KM interacted to their conserved domains. GPS-PUP predicted presence of pupylation sites within 15 proteins. Interactome also suggested that over expressed proteins interacted with each other and their partners except the hypothetical proteins. It is depicting that over expression of these proteins might not only neutralize/ modulate the effect of drug 
molecules but also are involved in various mechanisms, such as in mitigating the toxicity, repression of drug target and protein turnover to overcome the $\mathrm{AK}$ and $\mathrm{KM}$ resistance. We assume that cumulative effect of these over expressed proteins might be responsible to $\mathrm{AK}$ and $\mathrm{KM}$ resistance. These findings need further exploitation for the development of newer therapeutic agents or molecular markers which can directly be targeted to a gene/protein responsible for resistance so that an extreme condition like XDR-TB can be prevented and which could ultimately lead to exploration of newer therapeutics.

\section{AUTHOR CONTRIBUTIONS}

DS and DB design the concept of study, DS, ML, and RS involved in protein sample prepration and 2DGE, DS carried out the

\section{REFERENCES}

Andrusier, N., Nussinov, R., and Wolfson, H. J. (2007). FireDock: fast interaction refinement in molecular docking. Proteins 69, 139-159. doi: 10.1002/prot.21495

Beauclerk, A. A. D., and Cundliffe, E. (1987). Site of action of two ribosomal RNA methylases responsible for resistance to aminoglycoside. J. Mol. Biol. 193, 661-671. doi: 10.1016/0022-2836(87)90349-4

Beste, D. J., Espasa, M., Bonde, B., Kierzek, A. M., Stewart, G. R., and McFadden, J. (2009). The genetic requirements for fast and slow growth in mycobacteria. PLoS ONE 4:e5349. doi: 10.1371/journal.pone.0005349

Betts, J. C., Lukey, P. T., Robb, L. C., McAdam, R. A., and Duncan, K. (2002). Evaluation of a nutrient starvation model of Mycobacterium tuberculosis persistence by gene and protein expression profiling. Mol. Microbiol. 43, 717-731. doi: 10.1046/j.1365-2958.2002.02779.x

Bisht, D., Singhal, N., Sharma, P., and Venkatesan, K. (2007). An improved sample preparation method for analyzing mycobacterial proteins in two-dimensional gels. Biochemistry (Mosc). 72, 672-674. doi: 10.1134/S0006297907060119

Bradford, M. M. (1976). A rapid and sensitive method for quantitation of microgram quantities of protein utilizing the principle of protein-dye-binding. Anal. Biochem. 72, 248-254. doi: 10.1016/0003-2697(76)90527-3

Brodie, A. F., Kalra, V. K., Lee, S. H., and Cohen, N. S. (1979). Properties of energy-transducing systems in different types of membrane preparations from Mycobacterium phlei-preparation, resolution, and reconstitution. Methods Enzymol. 55, 175-200. doi: 10.1016/0076-6879(79)55024-1

Burns, K. E., Cerda-Maira, F. A., Wang, T., Li, H., Bishai, W. R., and Darwin, K. H. (2010). Depupylation of prokaryotic ubiquitin-like protein from mycobacterial proteasome substrates. Mol. Cell 39, 821-827. doi: 10.1016/j.molcel.2010.07.019

Canetti, G., Fox, W., Khomenko, A., Mahler, H. T., Menon, N. K., Mitchison, D. A., et al. (1969). Advances in techniques of testing mycobacterial drug sensitivity, and the use of sensitivity tests in tuberculosis control programmes. Bull. World Health Organ. 41, 21-43.

Darwin, K. H., Ehrt, S., Gutierrez-Ramos, J. C., Weich, N., and Nathan, C. F. (2003). The proteasome of Mycobacterium tuberculosis is required for resistance to nitric oxide. Science 302, 1963-1966. doi: 10.1126/science.1091176

de la Paz Santangelo, M., Gest, P. M., Guerin, M. E., Coinçon, M., Pham, H., Ryan, G., et al. (2011). Glycolytic and non-glycolytic functions of Mycobacterium tuberculosis fructose-1,6-bisphosphate aldolase, an essential enzyme produced by replicating and non-replicating bacilli. J. Biol. Chem. 286, 40219-40231. doi: 10.1074/jbc.M111.259440

de Souza, G. A., Arntzen, M. O., Fortuin, S., Schurch, A. C., Malen, H., McEvoy, C. R., et al. (2011). Proteogenomic analysis of polymorphisms and gene annotation divergences in prokaryotes using a clustered mass spectrometry-friendly database. Mol. Cell Proteomics 10:M110. doi: 10.1074/mcp.M110.002527

Eulenburg, G., Higman, V. A., Diehl, A., Wilmanns, M., and Holton, S. J. (2013). Structural and biochemical characterization of Rv2140c, a
2D-gel analysis, MALDI-TOF-MS and Bioinformatics analysis. $\mathrm{DS}, \mathrm{DB}, \mathrm{ND}$, and KV finalized the manuscript.

\section{FUNDING}

The research was financial supported by grant (No. 5/8/5/5/3/2011/-ECD-1) from ICMR, New Delhi. DS is ICMR-PDFs (ICMR, New Delhi).

\section{ACKNOWLEDGMENTS}

The authors are grateful to Director, NJIL and OMD for the support. The research was financial supported by grant (No. 5/8/5/5/3/2011/-ECD-1) from ICMR, New Delhi. DS is ICMRPDF (ICMR, New Delhi). We thank Mr. Jaypal for assistance. phosphatidylethanolamine-binding protein from Mycobacterium tuberculosis. FEBS Lett. 587, 2936-2942. doi: 10.1016/j.febslet.2013.07.038

Gorg, A., Obermaier, C., Boguth, G., Harder, A., Scheibe, B., Wildgruber, R., et al. (2000). The current state of two-dimensional electrophoresis with immobilized $\mathrm{pH}$ gradients. Electrophoresis 21, 1037-1053. doi: 10.1002/(SICI)15222683(20000401)21:6<1037::AID-ELPS1037>3.0.CO;2-V

Griffin, J. E., Gawronski, J. D., DeJesus, M. A., Ioerger, T. R., Akerley, B. J., and Sassetti, C. M. (2011). High-Resolution phenotypic profiling defines genes essential for mycobacterial growth and cholesterol catabolism. PLoS Pathog. 7:e1002251. doi: 10.1371/journal.ppat.1002251

Henriksson, L. M., Johansson, P., Unge, T., and Mowbray, S. L. (2004). X-ray structure of peptidyl-prolyl cis-trans isomerase A from Mycobacterium tuberculosis. Eur. J. Biochem. 271, 4107-4113. doi: 10.1111/j.1432-1033.2004.04348.x

Heym, B., Stavropoulos, E., Honoré, N., Domenech, P., Saint-Joanis, B., Wilson, T. M., et al. (1997). Effects of overexpression of the alkyl hydroperoxide reductase AhpC on the virulence and isoniazid resistance of Mycobacterium tuberculosis. Infect. Immun. 65, 1395-1401.

Hu, Y., Butcher, P. D., Mangan, J. A., Rajandream, M. A., and Coates, A. R. (1999). Regulation of hmp gene transcription in Mycobacterium tuberculosis: effects of oxygen limitation and nitrosative and oxidative stress. J. Bacteriol. 181, 3486-3493.

$\mathrm{Hu}, \mathrm{Y}$., and Coates, A. R. (2001). Increased levels of sigJ mRNA in late stationary phase cultures of Mycobacterium tuberculosis detected by DNA array hybridization. FEMS Microbiol. Lett. 202, 59-65. doi: 10.1111/j.15746968.2001.tb10780.x

Kruh, N. A., Troudt, J., Izzo, A., Prenni, J., and Dobos, K. M. (2010). Portrait of a pathogen: the Mycobacterium tuberculosis proteome in vivo. PLoS ONE 5:e13938. doi: 10.1371/journal.pone.0013938

Kumar, B., Sharma, D., Sharma, P., Katoch, V. M., Venkatesan, K., and Bisht, D. (2013). Proteomic analysis of Mycobacterium tuberculosis isolates resistant to kanamycin and amikacin. J. proteomics 94, 68-77. doi: 10.1016/j.jprot.2013.08.025

Lata, M., Sharma, D., Deo, N., Tiwari, P. K., Bisht, D., and Venkatesan, K. (2015b). Proteomic analysis of ofloxacin-mono resistant Mycobacterium tuberculosis isolates. J. Proteomics 127, 114-121. doi: 10.1016/j.jprot.2015.07.031

Lata, M., Sharma, D., Kumar, B., Deo, N., Tiwari, P. K., Bisht, D., et al. (2015a). Proteome analysis of ofloxacin and moxifloxacin induced Mycobacterium tuberculosis isolates by proteomic approach. Protein Pept. Lett. 22, 362-371. doi: 10.2174/0929866522666150209113708

Lee, H. N., Lee, N. O., Han, S. J., Ko, I. J., and Oh, J. I. (2014). Regulation of the ahpC gene encoding alkyl hydroperoxide reductase in Mycobacterium smegmatis. PLoS ONE 9:e111680. doi: 10.1371/journal.pone.0111680

Liu, Z., Ma, Q., Cao, J., Gao, X., Ren, J., and Xue, Y. (2011). GPS-PUP: computational prediction of pupylation sites in prokaryotic proteins. $\mathrm{Mol}$ Biosyst. 7, 2737-2740. doi: 10.1039/c1mb05217a 
Magnet, S., Courvalin, P., and Lambert, T. (2001). Resistance modulation cell division type efflux pump involved in aminoglycoside resistance in Acinetobacter baumannii BM4454. Antimicrob. Agents Chemother. 45, 3375-3380. doi: 10.1128/AAC.45.12.3375-3380.2001

Magnet, S., Smith, T. A., Zheng, R., Nordmann, P., and Blanchard, J. S. (2003). Aminoglycosides resistance resulting from tight drug binding to an altered aminoglycosides acetyl transferase. Antomicrob. Agents Chemother. 47, 1577-1583. doi: 10.1128/AAC.47.5.1577-1583.2003

Mashiach, E., Schneidman-Duhovny, D., Andrusier, N., Nussinov, R., and Wolfson, H. J. (2008). FireDock: A Web server for fast interaction refinement in molecular docking. Nucleic Acids Res. 36, W229-W232. doi: 10.1093/nar/gkn186

Maurya, V. K., Singh, K., and Sinha, S. (2014). Suppression of Eis and expression of Wag31 and GroES in Mycobacterium tuberculosis cytosol under anaerobic culture conditions. Indian, J. Exp. Biol. 52, 773-780.

Mawuenyega, K. G., Forst, C. V., Dobos, K. M., Belisle, J. T., Chen, J., Bradbury, E. M., et al. (2005). Mycobacterium tuberculosis functional network analysis by global subcellular protein profiling. Mol. Biol. Cell 16, 396-404. doi: 10.1091/mbc.E04-04-0329

Meena, L. S., Chopra, P., Bedwal, R. S., and Singh, Y. (2003). Nucleoside diphosphate kinase-like activity in adenylate kinase of Mycobacterium tuberculosis. Biotechnol. Appl. Biochem. 38, 169-174. doi: 10.1042/BA20020122

Nienaber, L., Cave-Freeman, E., Cross, M., Mason, L., Bailey, U. M., Amani, P. A., et al. (2015). Chemical probing suggests redox-regulation of the carbonic anhydrase activity of mycobacterial Rv1284. FEBS J. 282, 2708-2721. doi: $10.1111 /$ febs. 13313

Nikaido, H. (2003). Molecular basis of bacterial outer membrane permeability revisited. Microbiol. Mol. Biol. Rev. 67, 593-656. doi: 10.1128/MMBR.67.4.593656.2003

Palomino, J. C., Martin, A., Camacho, M., Guerra, H., Swings, J., and Portaels, P. (2002). Resazurin microtiter assay plate: simple and inexpensive method for detection of drug resistance in Mycobacterium tuberculosis. Antimicrob. Agents Chemother. 44, 2720-2722. doi: 10.1128/AAC.46.8.2720-2722.2002

Puckett, S., Trujillo, C., Eoh, H., Marrero, J., Spencer, J., Jackson, M., et al. (2014). Inactivation of fructose-1,6-bisphosphate aldolase prevents optimal cocatabolism of glycolytic and gluconeogenic carbon substrates in Mycobacterium tuberculosis. PLoS Pathog. 10:e1004144. doi: 10.1371/journal.ppat.10 04144

Quevillon, E., Silventoinen, V., Pillai, S., Harte, N., Mulder, N., Apweiler, R., et al. (2005). InterProScan: protein domains identifier. Nucleic Acids Res. 33, W116-W120. doi: 10.1093/nar/gki442

Raman, K., and Chandra, N. (2008). Mycobacterium tuberculosis interactome analysis unravels potential pathways to drug resistance. BMC Microbiology 8:234. doi: 10.1186/1471-2180-8-234

Rashid, M., Saha, S., and Raghava, G. P. S. (2007). Support vector machinebased method for predicting subcellular localization of mycobacterial proteins using evolutionary information and motifs. BMC Bioinformantics 8:337. doi: 10.1186/1471-2105-8-337

Rifat, D., Bishai, W. R., and Karakousis, P. C. (2009). Phosphate depletion: a novel trigger for Mycobacterium tuberculosis persistence. J. Infect. Dis. 200, 1126-1135. doi: 10.1086/605700

Schneidman-Duhovny, D., Inbar, Y., Nussinov, R., and Wolfson, H. J. (2005). PatchDock and SymmDock: servers for rigid and symmetric docking. Nucleic Acids Res. 33, W363-W367. doi: 10.1093/nar/gki481

Sharma, D., and Bisht, D. (2016). An efficient and rapid lipophilic proteins extraction from Mycobacterium tuberculosis H37Rv for two dimensional gel electrophoresis. Electrophoresis 37, 1187-1190. doi: 10.1002/elps.2016 00025

Sharma, D., Kumar, B., Lata, M., Joshi, B., Venkatesan, K., and Shukla, S., et al (2015b). Comparative proteomic analysis of aminoglycosides resistant and susceptible Mycobacterium tuberculosis clinical isolates for exploring potential drug targets. PLOS ONE 10:e0139414. doi: 10.1371/journal.pone. 0139414
Sharma, D., Lata, M., Faheem, M., Khan, A. U., Joshi, B., Venkatesan, K., et al. (2016). M.tuberculosis ferritin (Rv3841): potential involvement in Amikacin (AK) \& Kanamycin (KM) resistance. Biochem. Biophys. Res. Commun. 478, 908-912. doi: 10.1016/j.bbrc.2016.08.049

Sharma, D., Lata, M., Faheem, M., Khan, A. U., Joshi, B., Venkatesan, K., et al. (2015a). Cloning, expression and correlation of Rv0148 to amikacin \& kanamycin resistance. Curr. Proteomics 12, 96-100. doi: 10.2174/157016461202150903113053

Sharma, D., Shankar, H., Lata, M., Joshi, B., Venkatesan, K., and Bisht, D. (2014). Culture filtrate proteome analysis of aminoglycoside resistant clinical isolates of Mycobacterium tuberculosis. BMC Infect. Dis. 14(Suppl. 3):P60. doi: 10.1186/1471-2334-14-S3-P60

Sharma, P., Kumar, B., Singhal, N., Katoch, V. M., Venkatesan, K., Chauhan, D. S., et al. (2010a). Streptomycin induced protein expression analysis in Mycobacterium tuberculosis by two-dimensional gel electrophoresis \& mass spectrometry. Indian, J. Med. Res. 132, 400-408.

Sherman, D. R., Voskuil, M., Schnappinger, D., Liao, R., Harrell, M. I., and Schoolnik, G. K. (2001). Regulation of the Mycobacterium tuberculosis hypoxic response gene encoding alpha-crystallin. Proc. Natl. Acad. Sci. U.S.A. 98, 7534-7539. doi: 10.1073/pnas.121172498

Shevchenko, A., Wilm, M., Vorm, O., and Mann, M. (1996). Mass spectrometric sequencing of proteins from silver-stained polyacrylamide gels. Anal. Chem. 68, 850-858. doi: 10.1021/ac950914h

Shires, K., and Steyn, L. (2001). The cold-shock stress response in Mycobacterium smegmatis induces the expression of a histone-like protein. Mol. Microbiol. 39, 994-1009. doi: 10.1046/j.1365-2958.2001.02291.x

Singh, V., Mani, I., Chaudhary, D. K., and Somvanshi, P. (2011). The $\beta$-ketoacylACP synthase from Mycobacterium tuberculosis as potential drug targets. Curr. Med. Chem. 18, 1318-1324. doi: 10.2174/092986711795029636

Starck, J., Källenius, G., Marklund, B. I., Andersson, D. I., and Akerlund, T. (2004). Comparative proteome analysis of Mycobacterium tuberculosis grown under aerobic and anaerobic conditions. Microbiology 150, 3821-3829. doi: 10.1099/mic.0.27284-0

Stewart, G. R., Snewin, V. A., Walzl, G., Hussell, T., and Tormay, P. (2001). Overexpression of heat-shock proteins reduces survival of Mycobacterium tuberculosis in the chronic phase of infection. Nat. Med. 7, 732-737. doi: $10.1038 / 89113$

Vargas-Romero, F., Guitierrez-Najera, N., Mendoza-Hernández, G., OrtegaBernal, D., Hernández-Pando, R., and Castañón-Arreola, M. (2016). Secretome profile analysis of hypervirulent Mycobacterium tuberculosis CPT31 reveals increased production of $\mathrm{EsxB}$ and proteins involved in adaptation to intracellular lifestyle. Pathog. Dis. 74:ftv127. doi: 10.1093/femspd/ftv127

Welch, K. T, Virga, K. G., Whittemore, N. A., Ozen, C., Wright, E., Brown, C. L., et al. (2005). Discovery of non-carbohydrate inhibitors of aminoglycoside-modifying enzymes. Bioorg. Med. Chem. 13, 6252-6363. doi: 10.1016/j.bmc.2005.06.059

WHO Report (2015). Global Tuberculosis Control. Available online at: http://apps. who.int/iris/bitstream/10665/191102/1/9789241565059_eng.pdf

Zhang, H., Ouyang, H., Wang, D., Shi, J., Ouyang, C., and Chen, H. (2015). Mycobacterium tuberculosis Rv2185c contributes to nuclear factor-кB activation. Mol. Immunol. 66, 147-153. doi: 10.1016/j.molimm.2015.02.020

Conflict of Interest Statement: The authors declare that the research was conducted in the absence of any commercial or financial relationships that could be construed as a potential conflict of interest.

Copyright (C) 2016 Sharma, Lata, Singh, Deo, Venkatesan and Bisht. This is an open-access article distributed under the terms of the Creative Commons Attribution License (CC BY). The use, distribution or reproduction in other forums is permitted, provided the original author(s) or licensor are credited and that the original publication in this journal is cited, in accordance with accepted academic practice. No use, distribution or reproduction is permitted which does not comply with these terms. 